\title{
AN INVESTIGATION OF THE RADIAL DEPENDENCE OF THE GALAXY LUMINOSITY FUNCTION IN ABELL CLUSTERS
}

\author{
Phyllis M. Lugger \\ Department of Astronomy \\ Indiana University \\ Swain West 319 \\ Bloomington, IN 47405 \\ USA
}

ABSTRACT. The luminosity functions of inner and outer regions of six Abell clusters (A569, A1656, A2147, A2151, A2199, and A2634) were compared. These clusters have a single, reasonably symmetric central concentration of galaxies within the central Mpc. For three other clusters with irregular spatial distributions of galaxies (A779, A1367, and A2197) luminosity functions for high and low density regions were compared. For three of the clusters in the first group (A1656, A2147, and A2199) there is a deficit of bright galaxies, according to the Kolmogorov-Smirnov and Wilcoxon rank-sum nonparametric tests, in a region of radius $0.5 \mathrm{Mpc}$ about the cluster center compared to a concentric annular region with bounds of 0.5 and $1.0 \mathrm{Mpc}$.

\section{DATA SAMPLE}

Palomar 48 inch Schmidt R-band plates for nine clusters (A569, A779, A1367, A1656, A2147, A2151, A2197, A2199, and A2634) were digitized on the KPNO PDS and processed using the AUTOPHOT software as degcribed by Lugger (1986). Isophotal magnitudes to the $R=23 \mathrm{mag} \operatorname{arcsec}^{-2}$ isophote were measured for all objects in the scanned region. Stars were removed by a classifier based on image size relative to magnitude.

\section{LUMINOSITY FUNCTIONS}

An analysis of the galaxy surface density distribution in all nine clusters was carried out. For the six clusters (A569, A1656, A2147, A2151, A2199, and A2634) with a single, reasonably symmetric central concentration of galaxies within the central Mpc, luminosity functions of galaxies in inner and outer regions were compared. For the remaining three clusters (A779, A1367, and A2197), luminosity functions of galaxies in high and low density regions were compared. In order to compare galaxy magnitude samples without making any assumption about the form of the luminosity function, two nonparametric tests were used: the Kolmogorov-Smirnov two-sample test and the Wilcoxon rank-sum test. The figures show the three cases (A1656, A2147, and A2199) where a significarit difference was found between the luminosity functions in 
the two samples. These significant differences were found when comparisons were carried out both including and excluding the brightest galaxy (or first and second brightest galaxies for A1656 and A2147) in the cluster. The (a) figures are for the circular regions $0-0.5 \mathrm{Mpc}$ from the cluster centers and the (b) figures are for the annular regions $0.5-1.0 \mathrm{Mpc}$ from the centers. For these three cases there is a significant deficit of bright galaxies in the inner (a) region relative to the outer (b) region. The integrated luminosity functions, corrected for background, are indicated by histograms and the differential functions, corrected for background, are indicated by boxes with error bars. The crosses indicate the value of the differential function before background correction.
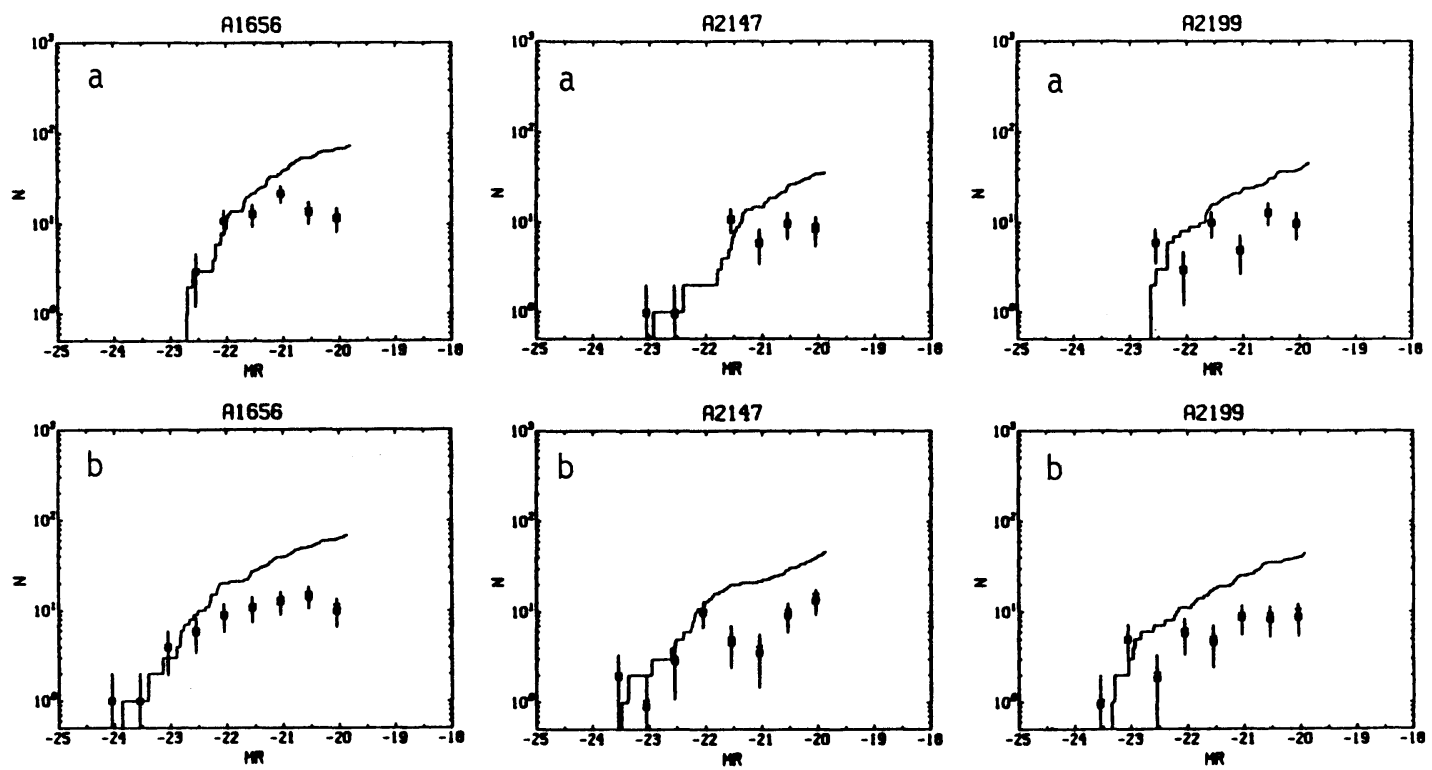

Figure 1. Luminosity functions for (a) inner and (b) outer regions.

\section{CONCLUSIONS}

The depletion of bright galaxies observed in the cores of A1656, A2147, and A2199 relative to outlying regions is an expected outcome of both galactic cannibalism (Hausman and Ostriker 1978) and tidal stripping. These dynamical processes, which have higher rates in higher density regions, affect the most massive galaxies to the greatest degree. The average surface densities within $0.5 \mathrm{Mpc}$ of the centers of all three clusters place them in the upper half of the range of central densities of the clusters studied here.

\section{REFERENCES}

Hausman, M.A. and Ostriker, J.P. 1978, Ap.J., 224, 320. Lugger, P.M. 1986, Ap.J., 303, 535. 